\title{
Erratum
}

\section{Causal structure of the thermal propagator in real-time formalisms}

\section{Lutz and T. Kunihiro}

Institute of Theoretical Physics, University of Regensburg, W-8400 Regensburg, Federal Republic of Germany

Z. Phys. C - Particles and Fields 49 (1991) 123

Equations (1.3), (2.4), (2.6) and (2.22) involve incorrect signs: In (1.3) the term $\Theta\left(-p_{0}\right) e^{x / 4}$ should be replaced by $-\Theta\left(-p_{0}\right) e^{x / 4}$. The rhs of $(2.4)$ should be multiplied by -1 . The factors $e^{\beta \mu / 2}$ and $-e^{-\beta \mu / 2}$ in (2.6) should be replaced by $-e^{-\beta \mu / 2}$ and $e^{\beta \mu / 2}$ respectively. Equation (2.22) should have read: $\sin \theta_{f}=\frac{\Theta(f(p)) e^{-x / 4}-\Theta(-f(p)) e^{x / 4}}{\sqrt{e^{x / 2}+e^{-x / 2}}}$

with $x=\beta\left(p_{0}-\mu\right)$. In the summary the phrase "in general incorrect" should be replaced by the phrase "not always correct". 